\title{
Traditional medicine information management
}

\author{
Aghebati Azita ${ }^{1, *}$, Safdari Reza ${ }^{1}$, Dargahi Hosein ${ }^{1}$, Gushe Gire Seid Ashrafoldin² \\ ${ }^{1}$ Paramedical Faculty, Tehran University of Medical Sciences, Tehran, Iran \\ ${ }^{2}$ Traditional Medicine Faculty, Tehran University of Medical Sciences, Tehran, Iran
}

\section{Email address:}

a_aghebati66@yahoo.com (A. Azita)

\section{To site this article:}

Aghebati Azita, Safdari Reza, Dargahi Hosein, Gushe Gire Seid Ashrafoldin. Traditional Medicine Information Management. American Journal of Health Research. Vol. 2, No. 2, 2014, pp. 43-48. doi: 10.11648/j.ajhr.20140202.12

\begin{abstract}
Objective: Given the increasing development of traditional medicine, the World Health Organization (WHO) has noted data management of traditional medicine and the need for acquiring the existing knowledge through the accurate exchange of information as well as preservation and protection of traditional knowledge resources. The use of traditional medicine in developing countries and developed countries is increasingly expanding. The purpose of this study is to developa traditional medicine information management processing. Methods: This is a review article which is carried out using books, articles, national and international journals as well as electronic resources and websites available on the Internet. Results: Despite the increasing development of traditional medicine, information has not been developed. Information development through web-based resources, proper communication with physicians and pharmaceutical packaging can lead to the development of information and quality of care. WHO has become a convenient and reliable source for information exchange. A global database of traditional medicine as well as a global database of medicinal herbs has been developed by WHO. The recommendations of WHO on documentation of traditional medicine include initial complaint recording, medical history, the findings of the physical examination, examination diagnosis and treatment plan, privacy and patient satisfaction, insurance and legal reports. Data elements should be standardized for comparison. The data can be transferred using electronic records. Furthermore, the record of data and assessment of patient's status will be done better. WHO published the international standard terminology that is the underlying data to better understanding of actions, research and training and developing a database to retrieve articles. The chapter 23 of the book, ICD 11, is developed tomake evidence-based traditional medicine, terminology, diagnosis classification, and designed interventions. It is also widely used in the International Classification of Traditional Medicine (ICTM) project which employs a wide range of traditional medicine knowledge worldwide. Conclusion: Given the development of traditional medicine, therapeutic interventions and medicinal complications of traditional and conventional medicine cause risks to patients. Development of reliable information and proper communication of patients and physicians will be helpful. Standard documentation, the global database and international classification and naming resources will integrate information and develop global statistics. Information development and information management process have an important role in improvement of patient care.
\end{abstract}

Keywords: Traditional Medicine, Database, Information

\section{Introduction}

The use of traditional medicine in developing countries and developed countries is increasingly expanding [1]. In recent years, the human approach to natural healing system and its reconstruction by the scientific community is due to several advantages including: 1-low cost health-care services, 2-easier access and 3- safety and efficacy [2, 3].

Budget allocated for traditional medicine is considerable so that 2.4 million dollars in Canada, 2.3 million dollars in UK and 2.2 million dollars in United States is spent on traditional medicine services. In 2000, the use of herbal medicine increased up to 60 billion dollars in the world.

The use of herbal products and dietary supplements in U.S. amounted to 17 billion dollars [4-7]. Of about 8 billion dollars medicinal costs in Germany, about 2.8 billion dollars is devoted to herbal medicine [8]. In Uganda, India and Ethiopia, the contribution of traditional medicine in primary health care is 60,70 and $90 \%$, respectively [9].

The lack of information and technical guidance prevents legislative progress, licensing and development of systems for monitoring and evaluation of complication in traditional 
medicine methods [10]. The World Health Organization's strategy for 2002-2005 has emphasized on data management of traditional medicine and the need for acquiring the existing knowledge through the accurate exchange of information as well as preservation and protection of traditional knowledge resources [11] and in2010-2011 on the development of research in whichinformation is fundamental.

This is a review article which is carried out using books, articles, national and international journals as well as electronic resources and websites available on the Internet and other documentary evidence in this regard. Purpose of this study is traditional medicine information management processing development.

\section{Finding}

\subsection{Traditional Medicine Definition}

World Health Organization (WHO) defined the traditional medicine as follows: "Traditional medicine is a general term which is referred to both traditional medicine systems like traditional Chinese medicine, Indian Ayurveda medicine, Greek-Arabic medicine as well as various forms of indigenous medicine [12] and is a comprehensive collection of knowledge, skills and experience based on the beliefs, assumptions and indigenous practices of various cultures that can be explained or not"[13].

\subsection{Availability Development of Traditional Medicine Information and Knowledge}

Despite increasing development of traditional medicine and expanding access to information and knowledge of traditional medicine, there is usually little information on the risk of adverse medicinal complications while concomitant use of herbal and chemical medicines [11]. Since opposed and harmful reactions to the proper communication between doctor and patient is of great importance to ensure the safety of the therapeutic interventions in conventional medical treatment and traditional medicine. Patient can get information in this regard to communicate more effectively [14]. Patients must provide the health centers with information on the type and name of drug, method of administration, the dosage, duration of drug use and the expected benefits to the drug [15].

Health care professionals can play an important role in reducing harm to patients treated by traditional medicine [16]. In USA(1990-1997), less than 40 percent of those who had used alternative traditional medicine therapies was brought to notify their physician in this regard [11].

Dissemination of information on a wide range of professional and commercial fields is necessary to ensure safe and effective educational and health standards of traditional medicine [17]. WHO has become as a reliable authoritative reference to share correct information on traditional medicine [11].
In 1999 -2000, a research on American patients clarified that more than half of them use the Internet to get medical information.

Free access to web-based resources in medicine can provide accurate and reliable information on the safety and effectiveness of regulatory policies, research and educational resources. Also, a quick update of the information is very important [17].

Information can help patients about the benefits and potential risks of the treatments as well as finding reliable resources. The product labels should be understandable for consumers. Elements of products information that usually is sufficient include: name, a list of active ingredients, dosage, precautions, method of administration, excessive dosage information, acute and even low side effects and complications, interventions, warnings, precautions and drug reactions, the use during pregnancy and lactation, expiration date and company information [12].

\subsection{Evidence-based Medicine (EBM)}

Evidence-based medicine is a type of medical knowledge and reasoning as a part of the evidence and documentation, not as a gold standard of clinical research and education in traditional medicine. Research is focused on the accuracy of diagnostic tests, predictive power of signs and safety and the therapeutic effects [19]. Research on the aspects of traditional medicine through clinical trials and successful measures are evidences that will result in medical and health alternatives [20].

\subsection{Traditional medicine Global Database}

The database has been developed using data and information collected through global survey by WHO. The database objectives include:

- Collecting and updating information on traditional medicine

- Sharing information and experiences to facilitate the establishment of appropriate national policies and regulations

- Monitoring the national progress in the field of traditional medicine as well as safe and effective use of herbal medicine

- Identifying the most troubled areas in the country and the support needed by the member states of the WHO

Frequent update of information in the future[21]

Traditional medicine has not reliable statistics on the disease, mortality, adverse complications and events. WHO has developed a national union consisted of 193 member states to gather health information. The collected data has been presented in the health information system. The World Health Organization is the only global representative that has carried out this process and collected data [22].

\subsection{Medicinal Herbs Global Database}

The medicinal herbs database of the WHO has been developed based on the database management and 
classification of medical herbs and data mining techniques for discovery of herbs reports, especially for finding out useful patterns among large different volumes of data. The medicinal systems should be linked to the global database to ensure the accessibility of international data.

Herbal Anatomical-Therapeutic Chemical Classification (HATC) is an initial classification of herbal products which the adverse reactions to its drugs have been reported in the WHO global database and then displayed in WHO Drug Dictionary. This classification is based on botanical science, pharmacology, phytochemicals, reviews and the use of old documents, the chemistry and EBM.[23]

Nowadays, the pharmacological research resulted in efforts in the documentation of knowledge and indigenous medical-scientific studies to improve health care as well as finding out pharmacological principles of indigenous treatment. The retrieve of research data on indigenous pharmacology is a challenging issue due to data within the journals from multiple disciplines or partial access to the studies. Scattered publications and different quality of data, incomplete databases, and institutions that do not have access to the wider scientific community restricts the scientists' access to research data. The advance in database technology to organize and analyze data is complex [24].

The reporting form of herbal therapy should be like other therapies. Countries have to edit their national reporting form to facilitate reporting interactions, herbal therapy interventions and other therapies.

The increase of data security includes who should process the data, for which aim, andto whomthe information should be transferred.[23]

\subsection{Documentation and Medical Records}

The WHO recommends following items for documentation and maintenance of the clinical records of traditional medicine:

Initial complaint recording, medical history, the findings of the physical examination, examination diagnosis and treatment plan, privacy and patient satisfaction, insurance and legal reports [25]

The requirements and content of medical records of traditional Chinese medicine

Identity information: include name, gender, age, marital status, nationality, race, occupation, workplace, home address, date of admission, history taking, phone number

2) the main complaint, 3) history of present illness, 4) past history, 5) personal history: personal habits, temperament, interests, 6) family history, 7)social history

This is the most important aspect of a patient's medical history so that it guides the medical team to make decisions based on the diagnostic results as well as planning for treatment [26].

8) the body structure: consciousness, mental condition, facial color and expressions, 9) section review, 10) reviewing tests, 11) diagnosis 10) 12) the treatment plan [27].

\subsection{Documentation of Treatment Plan}

The regulations of therapeutical plan emphasize on the patient's medical history, the patient's previous medical records, physical examinations, consultations, referrals and the use of other methods of treatment. The emphasized measures include:

1 - Evaluation: history, diagnosis and treatment and providing verbal informed consent for any traditional therapeutical plan.

2 - Therapeutical plan: doctors have to document a proper therapeutical with regard to individual health needs and the objectives specified in the plan.

3 - Periodic revision: doctors have to carry out a periodic documented revision of patient care at a reasonable frequency in order to progress therapeutical objectives and recording any new information on the patient's complaints and health care needs.

4 - Maintenance of medical records: doctors should maintain accurate and complete medical records of care provided to patients, especially assessments [28].

\subsection{Electronic Health Records}

Documentation of treatments in medical records leads to clarity of patient safety, the improvement of patient care, and research development.

Traditional medicine plays an important role in the health of patients. It should approach the appropriate consistent documentation to contribute to the assessment of the patient's status and his/her response to treatment. The data elements must be standardized as required so that the effect of different treatments in the cause of disruptions can be compared. If traditional medicine doctors improve to health electronic records, the data recording, information transferand assessment of the patient's status will be improved [29]. Digitization of health information provides opportunity for the input of traditional medicine information in computerized health information systems worldwide. The electronic records project will be followed in 2014-2015 [30].

\subsection{Traditional Medicine Terminology}

WHO International Standard Terminologies on Traditional Medicine in the Western Pacific Region: includes basic fundamental theories, diagnosis, diseases, various therapies including traditional Chinese medicine (TCM), Japanese medicine, Kampo, traditional Korean medicine (TKM) and traditional Vietnamese medicine (TVM) as the underlying data for better understanding of measures, research and education. This system has been used to retrieve various published articles [31].

Other resources include:

Wiseman's Practical Dictionary

The International Standard Chinese-English Basic Nomenclature of Chinese Medicine (32)

International classification standard for TCM doctor

International Standard Chinese-French Basic 
Nomenclature of Chinese Medicine

International Standard Chinese- Spanish Basic

Nomenclature of Chinese Medicine

International Standard Chinese- Portuguese Basic

Nomenclature of Chinese Medicine

These resources were developed by the World Federation of Chinese Medicine Societies (WFCMS). (33)

WHO International Standard Acupuncture Nomenclature [34]

\subsection{Classification and Coding of Traditional Medicine}

The codes of ICD-10 $10^{1}$ are used for homeopathy [35] and chiropractic [36]. Moreover, the problems caused by traditional medicine interventions are coded at ICD-10 u50 tier. The traditional treatments and application of different materials are wide-spreading, so their coding is difficult [37]. ICHI ${ }^{2} 2010$ includes traditional medicine interventions [38].

The efforts done by WPRO ${ }^{3}$ resulted in some information on the national classification of traditional medicine in China, Korea and Japan4 (ICTM-CJK). These activities consider traditional medicine as part of the future revisions of international classification of diseases (for example, ICD-11).[39] The chapter 23 of ICD- 11 develops a common language for the traditional medicine diagnosis. Furthermore, it helps the integration of traditional medicine and western medicine in public health [40] as well as integrity with accounting and system [41]. This source is used for development of traditional medicine based on evidence, terminology, classification of diagnosis and interventions. It also widely used in ICTM project which provides a wide range of traditional medicine knowledge worldwide [42]. International Classification of Traditional Medicine (ICTM) was proposed as an affiliated part of the WHO-FIC in 2006. One of the main issues of the project was introducing the international standard terminology and Accordance to ICD-10 [39]. Many experts from different countries collaborate in ICTM project [42]. The project was continued by development of three subgroups of acupuncture, herbal medicine and ICHI interventions [43]. It is expected that the ICTM project would develop the traditional medicine as mainstream medicine through recording traditional medicine terms in ontology software, establishing communication with the ICD and creating WHO-FIC $^{5}$ [39]. ICTM benefits include:

- The integration of traditional medicine with global standards and statistics and enhanced monitoring and public safety

- $\quad$ Increasing research on traditional medicine [42]

- Developing a policy for international database and a

1 International classification of disease and health related problem 10th revision

2International classification of health interventions

3 world federation of Chinese medicine societies

4Japan and Korea have their own traditional medicine adopted from ancient

China.

5WHO Family of International Classifications network for knowledge sharing [39]

- Reimbursement of more insurance to healers and traditional service providers

Producing data that can be used to assess the benefits, safety, applicability, costs as well as studying the role of traditional medicine in the prevention and treatment of diseases [44].

Traditional and conventional medicine systems are completely different [39]. Furthermore, translating the contents of traditional medicine into terms that can be understood and used by medical community is a challenging issue. The ICTM draft has been prepared [45].

\section{Conclusions}

Given the development of traditional medicine, the therapeutical interventions and medicinal side effects of traditional and conventional medicines cause risks to patients. The development of reliable information such as medicinal records, web-based resources and proper communication of patients and doctors will be helpful. Documentation standards for therapeutical plan, requirements and content of medical records, the standards for recording medical history and the benefits of electronic health record implementation of traditional medicine were discussed. WHO has developed international information by creating a global database of traditional medicine and integrated global statistics. Classification of medicinal herbs and WHO global database on medicinal herbs have been introduced. The indigenous pharmacological data have problems in standardization and data retrieve due to placement in multiple disciplines. The progress of technology is promising in facilitating data management. The international resources for naming and classification of traditional medicine interventions, Also international classification of traditional medicine which includes many benefits such as: integration with global standards and statistics, research development and delineating the role of traditional medicine in prevention and treatment of diseases has been introduced.

\section{References}

[1] Washington de who regional office for the Americas/ pan American health organization(working group ops/oms),World health organization traditional,complementary and alternative medicines and therapies., Washington dc ,1999

[2] Folder stiffen, magezine of payame- yonesco136,number 143

[3] jorjani seid esmaeil, zakhireye kharazmshahi ,Tehran, publisher by institute of iran culture: 1355

[4] White House commission on complementary and alternative medicine policy, find report, 2002 , capter 5: 69 .

[5] Who traditional medicine strategy 2002-2005 . Geneva , 2002 : 1-3, 43-47. 
[6] zad bakht mohamad, classification of herbal medicine,Tehran : teimoorxade publication, tabib published, 1378

[7] The promotion and development of traditional medicine report of a who meeting who report series, NO 622. Switzer land : who ; 1978

[8] Blumenthal M. editor the complete german commission $\mathrm{E}$ monographs - therapeutic guide to herbal medicines" , first ed , boston - Massachusetts , American botanical council , 1998: 17.

[9] Gyapony M. report on pre (2001 NOV ; 49(11) : 1577 testing of instrument of roll back material needs assessment in the dang me west district, Ghana .10 januray 1999 .

[10] Goldbeck wood S,dorozynski A,lie LG.complementary medicin is booming world wife.British medical journal $1996 ; 313: 131-33$

[11] Rezaie zade hosein, strategy of traditional medicine,tehran university of medical science publication 1384, Iranian traditional medicine research group, edited by dr naseri mohsen farmacologist of shahed university

[12] naseri mohsen, traditional medicine of iran and development by guidline of world health organization, daneshvar medicine magazine, university of shahed,

[13] International standard organization. Guideline of Action Plan on ISO/TC215-TCM Informatics. 2010; Available at: http://www.docstoc.com/docs/33247209/ISO-TC215-TCMInformatics (accessed in 16/4/2012)

[14] American cancer society. Guidelines for Using Complementary and Alternative Methods. 2011 http://www.cancer.org/Treatment/TreatmentsandSideEffects/ ComplementaryandAlternativeMedicine/guidelines-forusing-complementary-and-alternative-methods

[15] http://www.managingmenopause.org.au/management/othertherapies(accessed in 14/3/2012)

[16] http://www.cancervic.org.au/downloads/CISS factsheets/C AM_Info_Sheet_communwithpatients.pdf (accessed in $10 / \overline{5} / 201 \overline{2})$

[17] Gerard Bodeker, Fredi Kronenberg , A Public Health Agenda for Traditional, Complementary, and Alternative Medicine, 2002

[18] http://www.humanhealthproject.org/blog/?paged=2 (accessed in 12/4/2012)

[19] ministry of health \& social service, National guidline for medicine saftly surveillance, , therapetic information \& pharmacovigilance center, namibia ,africa, 2006

[20] unnikrishnan payyappallimana, Role of traditional medicine in primary health care, , Yokohama journal of social sciences , 2007

[21] world health organozation . National Policy on Traditional Medicine and Regulation of Herbal Medicines - Report of a WHO Global Survey ,2005

[22] Brenda Duran. First-Ever Information Standards For Traditional Medicine Go Online. 2011. available at: http://www.acupuncturetoday.com/mpacms/at/article.php?id $=32456($ accessed in $8 / 5 / 2012)$
[23] world health organization ,who guidlines on safty monitoring of herbal medicines in pharmacovigilance system, 2004 J.R. stepp, M.B. Thomas. Managing ethnopharmacological data: herbaria, relational database, literature. Available at: www.eolss.net/samplechapters/c03/E6-79-07.pdf(accessed in 6/2/2012)

[24] world health organization ,Benchmarks for training in traditional chinese medicine , 2010

[25] http://chinese-medicine.fozoe.com/?p=11 (accessed in $18 / 3 / 2012)$

[26] http://chinese medicine.fozoe.com/?p=1200(accessed in $17 / 2 / 2012$ )

[27] By Walter G. Mosher, J.D., M.H.A., LL.M. candidate, Complementary and Integrative Medicine: An Update for Texas Physicians

[28] Jennifer Hornung Garvin, Assessment across the Ages: Implications for the Electronic Health Record, Source: 2004 IFHRO Congress \& AHIMA Convention Proceedings, October 2004

[29] Marilyn Allen, Defining the Future of Traditional Medicine, 2010

[30] Seung-Hoon Choi and 11-Moo Chang, A Milestone in codyfing the wisdom of traditional oriental medicine:TCM, kampo, TKM,TVM_WHO international standardterminologies on traditional medicine in the Western Pasific Region , ,colllege of oriental medicine, 2009

[31] William Morris, Medical Epistemology: A Bias of Culture?, 20113

[32] $\mathrm{xu}$ chunbo,Innovation, standardization and $12^{\text {th }}$ five year plan of tcm,2011

[33] world health organization, A Proposed Standard International Acupuncture Nomenclature, Report of a WHO Scientific Group, 1991

[34] Available at: http://en.wikipedia.org/wiki/Health care in the United Sta tes . 2010 (accessed in 10/5/2012)

[35] John R. Bomar,Dynamic Chiropractic, 2012

[36] Roberta Pastore, Alessandro Campione, Bernardina Gonçalves , Use of ICD-10 for morbidity and mortality notification for in-patients, in recourse limited settings,2009

[37] Jean morie, Is the international classification of health care procedure (ICHI) a critical point for the implementation international case mix grouper?

[38] Zhong Xi Yi Jie He Xue Bao , Introduction of the World Health Organization project of the International Classification of Traditional Medicine ,Department of Traditional Chinese Medicine, Jinshan Hospital, Fudan University, 2011

[39] Kenji watanabe, integrating traditional medicine into ICD, 2012

[40] Eric Brand, Standards in Chinese Medicine: ICTM and ICD-11 Codes, 2011

[41] Brenda Duran. First-Ever Information Standards For Traditional Medicine Go Online. 2011. available at: 
http://www.acupuncturetoday.com/mpacms/at/article.php?id $=32456($ accessed in $12 / 6 / 2012)$

[42] world health organization, Annual report of the who collaboration center for the family of international classification for australia, Australian institue of health and welfare canberra
[43] Kylie O'Brien, Complementary and alternative medicine: the move into mainstream health care, Department of Medicine, Monash Medical School, Alfred Hospital, Prahran, VIC, Australia ,2004

[44] world health organization, WHO Developing New Traditional Medicine Classification, 2011 